\title{
CONOCIMIENTO, PRÁCTICA Y PERCEPCIÓN DE ENFERMERAS RESPECTO A TELE-ENFERMERÍA COMO ESTRATEGIA DE CONTINUIDAD DEL CUIDADO
}

\author{
KNOWLEDGE, PRACTICE AND PERCEPTION OF NURSES REGARDING \\ TELE-NURSING AS A STRATEGY FOR THE CONTINUITY OF CARING \\ CONHECIMENTO, PRÁTICA E PERCEPÇÃO DAS ENFERMEIRAS COM RELAÇÃO \\ À TELE-ENFERMAGEM COMO ESTRATÉGIA DE CONTINUIDADE DO CUIDADO
}

\author{
Lucía Carvajal Flores \\ Universidad de Costa Rica. San José, Costa Rica. \\ lucf18@gmail.com \\ ORCID: 0000-0001-8313-2508 \\ Leidy Vásquez Vargas \\ Universidad de Costa Rica. San José, Costa Rica. \\ ORCID: 0000-0001-6393-1724
}

DOI: http://dx.doi.org/10.22235/ech.v5i2.1284

Recibido: 23/05/2016

Aceptado: 09/10/2016

\section{RESUMEN}

La Tele-Enfermería es el cuidado de Enfermería a la distancia, que se realiza mediante el uso de herramientas tecnológicas. Los profesionales que la utilizan planifican, intervienen y evalúan los resultados de la atención utilizando las tecnologías de la información y comunicación. El objetivo de este estudio fue determinar el conocimiento, práctica y percepción de enfermeras que laboran en un hospital público clase A de Costa Rica, respecto a la Tele-Enfermería como estrategia de continuidad del cuidado. Se optó por una investigación cuantitativa exploratoria transversal; se implementó un cuestionario tipo entrevista previamente validado con una consistencia interna de 0.96 , conformado por 38 preguntas abiertas y cerradas. La población fue de 251 profesionales de enfermería que laboran dentro de dicha institución de salud y la muestra seleccionada fue de 104. Los datos obtenidos fueron tabulados, procesados y analizados mediante el uso del paquete estadístico SPSS versión 22 y la Escala Aditiva. Se identificó que la mayor parte de las participantes tenían entre 26 y 45 años y habitaban dentro del Gran Área Metropolitana. Una minoría conocía el término Tele-Enfermería previo al cuestionario, la mayoría sí había implementado prácticas relacionadas con tecnología por lo que la mayor parte de las personas entrevistadas tuvieron una perspectiva muy favorable respecto a la temática. Este artículo es el primero de su índole a nivel costarricense; intenta abordar un tema con muchos vacíos pero con múltiples beneficios para la salud nacional y global. A la vez, busca generar diálogo con la comunidad internacional sobre nuevas formas de llevar cuidados aprovechando los avances de la globalización y demostrando que la enfermería es una profesión capaz de modernizarse y romper barreras geográficas y temporales.

Palabras clave: Enfermería, Atención de Enfermería, Informática Médica, Tecnología, Consejería a Distancia.

\section{ABSTRACT}

Tele-Nursing is nursing care at a distance, which is done using technological tools. The professionals who use Tele-Nursing plan, intervene and evaluate the results of nursing care using information and communication technologies. The objective of this study was to determine the knowledge, practice and perception of nurses working in a Class A public hospital in Costa Rica, regarding Tele-Nursing as a strategy of continuity of care. A cross-sectional exploratory quantitative research was carried out using a pre-validated interview questionnaire with an internal consistency of 0.96 , consisting of 38 open and closed questions. The population was 251 nursing professionals working within this health institution and the selected sample was 104. The data obtained was processed and analyzed using the statistical package SPSS version 22 and the Additive Scale. The majority of the participants were between 26 and 45 years old and lived within the Greater Metropolitan Area. A minority knew the term Tele-Nursing prior to the questionnaire and most had implemented technology-related practices; therefore most of the people interviewed had a very favorable perspective on the subject. This article is the first of its kind at the Costa Rican level; it attempts to address a topic with many unknowns but with multiple benefits for national and global health. At the same time, it seeks to generate a dialogue with the international community on new ways of nursing care by taking advantage of the advances of globalization and demonstrating that nursing is a profession capable of modernizing and breaking geographical and temporal barriers.

Keywords: Nursing, Nursing care, Informatics, Technology, Medical Informatics, Distance Counseling. 


\section{RESUMO}

A tele-enfermagem é o cuidado de Enfermagem à distância, realizado mediante o uso de ferramentas tecnológicas. Os profissionais que utilizam esta ferramenta planejam, intervém e avaliam os resultados da atenção utilizando as tecnologias da informação e comunicação. O objetivo deste estudo foi determinar o conhecimento, prática e percepção das enfermeiras que trabalham em um hospital público classe A de Costa Rica, com relação à tele-enfermagem como estratégia de continuidade do cuidado. Optou-se pela pesquisa quantitativa exploratória transversal, aplicando um questionário do tipo entrevista, previamente validado, com uma consistência interna de 0,96, conformado por 38 perguntas abertas e fechadas. A população foi de 251 profissionais da enfermagem que trabalham dentro dessa instituição de saúde, e a amostra selecionada foi de 104. Os dados obtidos foram tabulados, processados e analisados mediante o uso do pacote estatístico SPSS e a Escala Aditiva. Identificou-se que a maior parte das participantes tinha entre 26-45 anos, e morava dentro da Grande Área Metropolitana. A minoria conhecia o termo tele-enfermagem antes do questionário; a maioria já havia implementado práticas relacionadas com a tecnologia, e a maior parte das pessoas entrevistadas teve uma perspectiva muito favorável com relação à temática. Este artigo é o primeiro do seu tipo a nível costarriquenho e tenta abordar um tema com muitos vazios, mas com múltiplos benefícios para a saúde nacional e global. Ao mesmo tempo, procura gerar diálogo com a comunidade internacional sobre novas formas de prestar cuidados aproveitando os avanços da globalização, demonstrando que a enfermagem é uma profissão capaz de se modernizar e vencer barreiras geográficas e temporais.

Palavras-chave: Enfermagem, Cuidados de Enfermagem, Informática Médica, Tecnologia, Aconselhamento à Distância.

\section{INTRODUCCIÓN}

La salud no es solamente la ausencia de afecciones o enfermedades sino que, como expresa la Organización Panamericana de la Salud en el 2007, es un fenómeno con implicaciones sociales, económicas y políticas, en la que influyen determinantes sociales y tiene prerrequisitos (1-4). Es un concepto complejo que a la fecha no incluye en sus definiciones como un factor de impacto a la tecnología o el acceso a ella. Algunos autores han clasificado el acceso a la información como determinante social de la salud y otros consideran su uso como una forma de traspasar barreras hospitalarias (5-6). Las Tecnologías de la Información y la Comunicación, conocidas como TIC, han surgido y generado un impacto a nivel global nunca antes imaginado; se cree que la evolución de las mismas proseguirá y muy probablemente se acelerará. Esta es una temática inherente al mundo moderno y se ha permeabilizado a todos los campos de la sociedad, siendo la salud parte de ello $(7,8)$.

Las TIC representan una oportunidad para ofrecer cuidados; incluso desde 1990 la OPS menciona la importancia de aplicar estas tecnologías en las instituciones de salud y se puede mostrar en la profesión de enfermería, la cual ha empezado a implementar dispositivos electrónicos para mejorar el cuidado de la población y ha producido nuevos conceptos como el término Tele-Enfermería (9).
Bartz y otros comentan que el prefijo tele significa distancia; por consiguiente, mencionan que la Tele-Enfermería se refiere al cuidado de Enfermería a la distancia. A la vez comentan dentro de su artículo que se utiliza tanto para ofrecer cuidados como para llevar a cabo la práctica de enfermería y la Asociación Americana de Enfermeras (ANA) la ha definido como un subconjunto de la telesalud, en el que la atención se centra en la práctica de la profesión específica: la Enfermería $(10,11)$.

En 2008 el Consejo Internacional de Enfermeras (CIE) en su documento establece: "Servir a la comunidad y garantizar la calidad: las enfermeras al frente de la atención primaria de salud", expone el término Tele-Enfermería y la da a conocer como un área de gran interés, que permite mejorar el acceso a las cuidados y mejorar la calidad y continuidad de los mismos independientemente de las distancia (11). Junto con lo anterior, Bartz en una conferencia plantea los "US Telenursing Collaborative Efforts to Achieve Goals", donde se muestran los servicios en línea con que ya se cuentan alrededor del mundo relacionados con Tele-Enfermería y las metas propuestas por el CIE respecto al tema (10). Esta es una estrategia aplicada en países como Colombia, Nueva Guinea, Canadá, Brasil, África, Finlandia, España entre otras regiones, para brindar cuidados específicos, consultas, capacitaciones y educación de forma continua y accesible, incluso, para quienes la distancia obstaculiza el acceso a los centros de salud (11-16).

Las enfermeras deben ser, según Arredondo y Siles, “...el puente que une la tecnología con la atención de salud humana y tiene la responsabilidad de mantener la humanidad en los medios ambientes tecnológicos...", esta capacidad es una particularidad de la Enfermería, ya que la esencia de la profesión es el cuidado del ser humano, es decir, es una ciencia enfocada en la persona y si bien se pueden generar cambios en los procesos de gestión clínicos y administrativos a partir de medios tecnológicos, la atención del profesional de enfermería debe ejercerse siempre con humanismo y ética (17).

Costa Rica en los últimos años ha mejorado en el área de las telecomunicaciones. Cordero menciona que según el Índice de Desarrollo de las Tecnologías de Información y la Comunicación (UDI) el país subió cinco posiciones (18). En relación con lo mencionado se considera la viabilidad de implementar la Tele-Enfermería como una estrategia de continuidad de cuidados a la distancia, servicio que actualmente no se ofrece a nivel costarricense y es un tema poco estudiado por parte del sector salud.

Por ello, para dar un primer paso se considera importante incluir a los profesionales en la temática y de esta forma identificar ¿cuál es conocimiento, práctica y percepción de las enfermeras que laboran en un hospital público clase $A$, en relación a la Tele-Enfermería como una estrategia de continuidad para el cuidado?

\section{METOdOLOGÍA}

Se trata de un estudio exploratorio transversal, realizado a un grupo de 104 enfermeras de un hospital público clase A de Costa Rica, ubicado dentro del Gran Área Metropolitana (GAM). La 
población fue 251 profesionales, la totalidad del cuerpo enfermero del lugar. El muestreo utilizado fue por conveniencia, en el que se contemplaron los siguientes criterios de inclusión: estar contratados como enfermera profesional y que estuvieran laborando el día, el turno y la hora en que las investigadoras asistían al centro de salud. Por eso la aplicación del instrumento se realizó a un total de 104 personas. Los datos fueron recolectados durante el primer semestre del 2014.

Las entrevistas se desarrollaron mediante un cuestionario realizado por las investigadoras; posteriormente se validó por medio de 3 tipos de evidencia: validez de contenido, de constructo y de expertos. La consistencia interna del instrumento obtuvo una confiabilidad global $\alpha$ Cronbach de 0.96 . Por eso el instrumento constó de 38 ítems los cuales tenían preguntas abiertas y cerradas. El instrumento recogió información sobre las cuatro variables (sociodemogáfica, conocimiento, práctica y percepción), que permitió responder a los objetivos del estudio (Ver anexo 1).

Para el análisis de la información se consideró primero indagar contenido adjunto dentro de un marco referencial previamente construido mediante una búsqueda exhaustiva de referencias bibliográficas relacionadas con la temática. Estas son: antecedentes, definiciones, noticias, artículos científicos, documentos, libros, proyectos implementados, entre otros. En segundo lugar los datos recolectados en los cuestionarios se ordenaron y procesaron utilizando el Paquete Estadístico para Ciencias Sociales versión 22 (SPSS) y para la variable de percepción se sumaron la totalidad de las respuestas mediante la Escala Aditiva, para identificar si la percepción que se tiene respecto al tema es positiva o negativa. En tercer lugar se realizó el análisis de los resultados de la investigación, considerando los datos finales obtenidos por medio de SPSS y la Escala Aditiva la cual se obtiene con "las puntuaciones de las escalas Likert (...) sumando los valores alcanzados respecto de cada frase (19).

Para la realización del estudio se obtuvo autorización del centro de salud con las autoridades y el "Comité de Investigación y Ética" pertinente y se realizó un consentimiento verbal y escrito a las participantes. También se ha garantizado el anonimato de las personas participantes y la confidencialidad de la información; además se veló por el cumplimiento de los principios bioéticos de la autonomía, beneficencia, no maleficencia y justicia.

\section{RESULTADOS}

La mayoría de los participantes se encontraban en un rango de edad 26 a 45 años, un 68,3\% fueron mujeres, 97,1\% de nacionalidad costarricense y la totalidad de entrevistados habitaban dentro del Gran Área Metropolitana. Los puestos de trabajo de las participantes fueron diversos coordinadores, supervisores y enfermeras del rango 1 al 5. Además laboraban dentro de servicios como: cirugías, consulta externa, urgencias, dirección de Enfermería, ginecoobstetricia, hemodiálisis, maternidad, medicina, neonatología, neurociencias, quimioterapia, consulta de ostomizados, salud mental y psiquiatría, recuperación, unidad de cuidado intensivo, sala de operaciones, programa del alta hospitalaria, entre otros.
Respecto al conocimiento de las enfermeras participantes en relación a la Tele-Enfermería se identificó que un 52\% habían escuchado sobre las TIC, atribuyendo haber oído el término principalmente en sitios como: $29 \%$ en la universidad, $26 \%$ en el lugar de trabajo y $24 \%$ en un congreso. Para identificar si las participantes contaban con algún conocimiento previo de computación, telemática y cursos online, se les preguntó si habían llevado algún curso y/o capacitación al respecto, resultando que un $69 \%$ había llevado un curso de computación, un $19 \%$ un curso online, un $4 \%$ cursos de telemática y un $25 \%$ ninguno de los anteriores.

En relación al conocimiento sobre algunas terminologías utilizadas en cibersalud, los resultados muestran que un $68 \%$ sabían sobre Tele-Medicina, un 26\% de Tele-Enfermería, un 13\% sobre E-Learning y Tele-Salud, un 10\% sobre Tele-Cuidado, $9 \%$ Tele-Farmacia, $5 \%$ Tele-Psiquiatría, $1 \%$ E-health y $20 \%$ no había escuchado ninguno de los términos consultados. A la vez un $63 \%$ afirmó conocer con certeza que las TIC son aplicadas al área de salud en Costa Rica mientras un 37\% negó conocer al respecto.

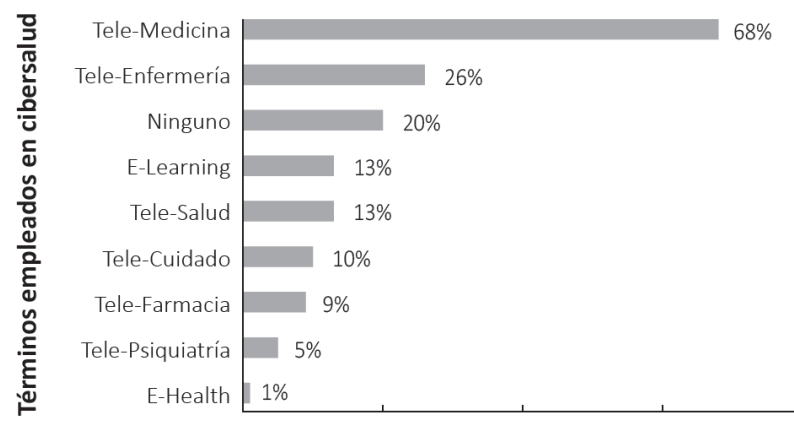

Porcentaje de enfermeras(os) que los conocen

Fuente: Elaboración propia (2016)

Gráfico 1 - Términos sobre cibersalud conocidos por el personal de enfermería de un hospital público clase A de Costa Rica, I semestre 2014.

En lo relacionado a la práctica de enfermeras participantes respecto a la Tele-Enfermería se identificó que la mayoría utiliza aplicaciones electrónicas (apps) dentro de sus dispositivos electrónicos, principalmente las relacionadas con: fotografías y videos $(88 \%)$, música $(81 \%)$, entretenimiento $(80 \%)$, redes sociales (79\%), educación (78\%), navegación (76\%), medicina (75\%), libros (72\%) y noticias (69\%). Asimismo, alrededor de la mitad utiliza aplicaciones de salud-forma (59\%) y clima (52\%), menos de la mitad de comida-bebida (44\%) y estilos de vida (39\%). Finalmente, deportes (28\%), finanzas (21\%) y negocios $(17 \%)$ son las menos utilizadas.

En lo que respecta al lugar de trabajo se observó que el $87 \%$ utiliza el teléfono fijo y el $53 \%$ la computadora. Cabe mencionar, que un $28 \%$ expresaron no tener computadora en su servicio o departamento hospitalario; además quienes conocían de su existencia y no la utilizaban, expusieron como los principales motivos para no hacerlo: $10 \%$ por la lentitud de la máquina y $9 \%$ por pasar ocupada por otros profesionales.

Durante la jornada laboral, las participantes utilizan el teléfono fijo, principalmente para comunicarse con otros servicios y de- 
partamentos dentro del mismo centro hospitalario (83\%), para comunicarse con otros profesionales del área de la salud (78\%), con la familia o responsable de un usuario (77\%) y lo emplean en menor medida para comunicarse con un usuario respecto a su estado de salud (57\%).

Por otro lado, el ordenador es aprovechado en el trabajo mayoritariamente para conocer resultados de laboratorio y exámenes médicos (49\%), utilizándolo raramente para comunicarse con un usuario respecto a su estado de salud (3\%) y con la familia o responsable de un usuario (3\%). En lo que respecta al uso de algún medio de comunicación para informarse sobre la condición de salud de un usuario que se encuentra a distancia, un $82 \%$ afirmó que utiliza una llamada telefónica, $29 \%$ mensaje de texto, $17 \%$ ninguno de los medios consultados, $16 \%$ correo electrónico, $10 \%$ chat y $3 \%$ vídeo llamada. (Ver gráfico 3 ).

Referente a la percepción de los participantes respecto al tema de la Tele-Enfermería, el $81 \%$ indicó estar totalmente de acuerdo con las TIC como una herramienta funcional en el campo de la salud. Además, resulta importante considerar que el $83 \%$ afirmó estar "totalmente de acuerdo" en la aplicación de TIC en Enfermería, lo cual es muy positivo, ya que la implementación de nuevas prácticas para el cuidado son vitales. Se evidenció que el $63 \%$ respondió estar "totalmente de acuerdo" y un $26 \%$ "de acuerdo" en que es posible dar continuidad de cuidados a la distancia por medio de dispositivos electrónicos.

Como resultado de la aplicación de la escala aditiva se determina que los participantes poseen una percepción positiva de la Tele-Enfermería como una estrategia de continuidad de cuidado.

$82 \%$

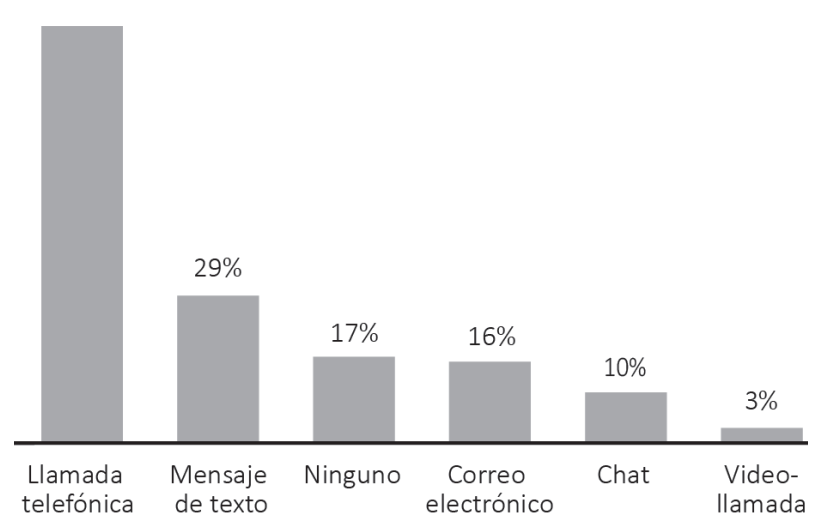

Fuente: Elaboración propia (2016)

Gráfico 2 - Medios de comunicación utilizados por la población de enfermería de un hospital público clase A de Costa Rica para informarse sobre un usuario(a) a la distancia, I semestre 2014.

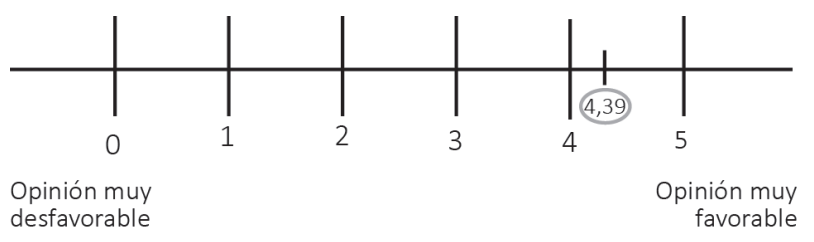

Fuente: Elaboración propia (2016)

Figura 1 - Puntuación de la Escala de Likert según la percepción de la población de enfermería de un hospital público clase A de Costa Rica con respecto a la Tele-Enfermería, I semestre 2014.

\section{DISCUSIÓN}

En el 2001 la Organización Mundial de la Salud analiza la evolución que ha tenido la tecnología y sus tendencias en el sector salud, aborda sobre la implementación que se está dando y la situación en la que vive América Latina y el Caribe, además de las oportunidades que éste le provee a dichos sectores (20). Esta temática es de interés para enfermería, aunque es necesario conocer en primer lugar el conocimiento, práctica y percepción de quienes ejercen la profesión en el día a día, para poder generar toma de decisiones desde las diferentes posiciones estratégicas del campo sanitario tanto a nivel de Costa Rica como a nivel internacional.

En un estudio se demuestra que la mayor parte de las personas entrevistadas conocían y estaban familiarizadas con la temáti$\mathrm{ca}$, lo cual se relaciona con el hecho que la mayoría de personas participantes habían nacido posteriormente a la década de los setenta, período conocido como "Era de la comunicaión y las telecomunicaciones" (7). A la vez, la totalidad de las personas entrevistadas eran habitantes del GAM, zona de Costa Rica que ofrece gran facilidad de acceso a la tecnología y cobertura de internet (21).

En la Cumbre Mundial de la Sociedad de la Información, realizada en Ginebra en el 2013, se expone el compromiso de hacer de la sociedad de la información un medio para lograr el bienestar de las personas a través del acceso a la información, al conocimiento y a las TIC (22). Sin embargo, en esta investigación un $48 \%$ de los profesionales afirmaron no haber escuchado sobre las TIC con anterioridad, lo que además es coherente con lo que Orem afirma desde 1983, que la Enfermería y su sistema de educación se han visto afectados por la falta de formalización de tecnología esencial para su práctica. Esto lleva a confirmar la necesidad de incluir estas temáticas dentro de la formación formal y no formal universitaria (23). Por otro lado, las personas que sí habían escuchado lo habían hecho en lugares y eventos de formación académica y profesional.

La construcción de una Sociedad de la Información centrada en la persona es un esfuerzo conjunto que necesita la cooperación y la asociación de todas las partes interesadas (24). En los resultados se encontró que existen profesionales con conocimientos en temas como computación, telemática y cursos online, por lo que si estas habilidades se complementaran con estudio, capacitaciones, esfuerzo, interés y apoyo de las instituciones gubernamentales y no gubernamentales, la Tele-Enfermería podría convertirse en un motor propulsor para agilizar los trámites de los servicios hospitalarios, fortalecer una mejor atención al público y generar grandes oportunidades para el avance de la profesión y la calidad de vida de las personas.

Las TIC han buscado optimizar, agilizar e impulsar el progreso del sector salud e incluso en las últimas cuatro décadas la evolución de las aplicaciones de TIC han ofrecido una gran variedad de recursos y con ellos se han forjado terminologías nuevas para referirse a su uso (15). En los resultados se identificó que el término que conocían más participantes (68\%) fue el de Tele-Medicina; esto se debe a que la Caja Costarricense de Seguro Social (CCSS) comenzó a implementar la telemedicina desde hace más de trece años (25). 
Si bien las TIC han estado presentes en algunos quehaceres de la Enfermería, no se ha empleado el término a nivel de la CCSS ni de Costa Rica para referirse a la Tele-Enfermería como el cuidado de Enfermería a la distancia (10). En este sentido, González expone que en el país no existe una especialización en Enfermería Informática y no se ha proyectado a nivel nacional capacitaciones $u$ oportunidades para dar a conocer el tema, por lo que únicamente el $26 \%$ de los entrevistados había escuchado sobre dicho término (26). Esta falta de divulgación de información también se ve reflejada en el poco conocimiento sobre las diversas terminologías de otras disciplinas, como E-learning, Tele-Salud, Tele-Cuidado, Tele-Farmacia, Tele-Psiquiatría, E-health, escuchadas solamente por la minoría de los participantes.

A nivel global se observa este fenómeno: al comenzar el siglo XXI no más del $6 \%$ de la humanidad accedía a Internet y a finales de 2012 prácticamente un $35 \%$ de la humanidad se encontraba conectada a Internet (27). Este servicio brinda la oportunidad de descargar "apps". Una "app", o "aplicación", es un programa informático que permite que el usuario lleve a cabo una o varias operaciones a través de dispositivos móviles. Lo más importante según Gardner y Davis es que son rápidas, satisfacen una demanda y el fin buscado es que las aplicaciones logren proporcionar al ser humano todo cuanto pueda necesi$\operatorname{tar}(28)$.

Mediante los resultados se logra identificar que las participantes implementan dentro de su vida cotidiana diferentes tipos de "apps" en sus dispositivos electrónicos, ejemplificando con ello la influencia que las mismas tienen en todas las áreas de la sociedad y han logrado causar implicaciones globales e individuales, modificando la forma de comunicación, la transmisión, el procesamiento y el almacenamiento de información.

Relacionado con el uso de dispositivos como el teléfono fijo y la computadora en el lugar de trabajo, los datos obtenidos en la investigación manifiestan la necesidad de buscar estrategias que permitan implementar las TIC para ofrecer continuidad de cuidados a la distancia, efectiva y accesible a la mayoría de los individuos y sus familias. Los resultados demuestran que, a pesar de las ventajas que ofrecen las computadoras, muchas empresas no las están aprovechando plenamente y en muchas otras ni siquiera la utilizan (29). Sin embargo la computadora dentro del ámbito hospitalario ofrece múltiples funciones: agilizar trámites hospitalarios, mejorar la calidad de atención, dar educación a la distancia, mejorar la adherencia terapéutica, ofrecer un seguimiento a los usuarios luego del egreso, entre muchas otras.

No obstante, a nivel costarricense y específicamente en el ámbito hospitalario queda muchísimo trabajo para incorporar las TIC en sus procesos productivos, desde invertir en equipo y capacitación hasta cambios en el modelo institucional; sin embargo, como expone el Programa Sociedad de la Información y el Conocimiento (PROSIC), es importante considerar que es un proceso gradual que puede tomar algún tiempo pero que debe promoverse desde ya (29).
Las TIC avanzan cada día con nuevas ideas de dispositivos, de negocios, de aplicaciones y de usos que van generando grandes beneficios para la salud pública y para el resurgimiento de una profesión que en algunos países del mundo necesita renovar su identidad, sus ideas y la forma de acercarse a las comunidades, los individuos y familias. Por ello no es de extrañarse que la percepción de la mayoría de los participantes dentro de esta investigación hayan tenido una opinión muy favorable de la Tele-Enfermería como una estrategia de continuidad de cuidado.

\section{CONSIDERACIONES FINALES}

La Enfermería debe buscar enfocarse en ser una profesión que cambia y se adapta a la globalización. Este camino debe darse en todos los planos, empezando por las mallas curriculares, que muchas veces son obsoletas. Si el mundo cambia, las poblaciones, sociedades y formas de vida también lo hacen, por lo que la profesión también debe irse transformando para lograr que los servicios ofrecidos sean adecuados y actualizados a las personas y la familia moderna.

Proyectos como un departamento de Tele-Enfermería podría generar grandes beneficios para la salud pública de Costa Rica y el mundo, como por ejemplo: brindar un seguimiento luego del egreso hospitalario, acortar distancias, disminuir reingresos hospitalarios, mejorar la adherencia terapéutica, evitar complicaciones de salud mediante una mejor comunicación, ofrecer cuidado personalizado, promover la participación de la población en sus decisiones respecto a temas de salud, impulsar la educación a distancia, promover la actualización e investigación continua de los profesionales y proyectar aún más la profesión de enfermería a la sociedad global. Tal como comenta Jardines "las tecnologías debieran jugar un papel determinante en la noble aspiración de lograr salud para todos" (30). Este estudio presentado demuestra que la percepción de los participantes respecto al uso de Tele-Enfermería como una estrategia de continuidad de cuidados es muy favorable. Esto es fundamental, ya que los profesionales actuales tienen la apertura y la disposición necesaria para emprender el desafío de buscar formas de implementar los avances tecnológicos dentro de sus campos.

Las crecientes expectativas en el uso de las TIC ponen de manifiesto la necesidad de que los profesionales de Enfermería estén formados en su uso y en su conocimiento para poder dar respuesta a las necesidades y demandas de la sociedad (22). Sin embargo, se tiene claro que estas transformaciones no estarán exentas de personas, empresas e instituciones que tengan resistencia al cambio, pero eso es parte del reto por enfrentar, así como un día no se vislumbraba la posibilidad de la existencia de un celular o de Internet hoy no se proyecta el futuro $\sin$ estas facilidades.

\section{LIMITACIONES DEL ESTUDIO}

Por último, cabe destacar que para la elaboración del estudio se tuvo una serie de limitantes en relación a la metodología, ya que inicialmente se consideró utilizar una muestra probabilística con selección aleatoria. Sin embargo a pesar de contar con la lista del total de enfermeras y utilizar la tabla de números 
aleatorios para realizar la selección, no fue posible aplicar dicho muestreo porque algunas de las personas seleccionadas no se encontraban en su lugar de trabajo por motivo de incapacidad, vacaciones, cambios de horario o día libre asignado, siendo este tipo de selección un riesgo para la conclusión oportuna del estudio. Por tanto, se reformuló el tipo y selección de la muestra, la cual fue descrita anteriormente dentro de la metodología de la investigación. A pesar de dichas limitaciones, este estudio tiene grandes fortalezas, ya que genera un aporte para el sistema nacional de salud al documentar aspectos relevantes que pueden tener la clave para lograr el acceso y la cobertura universal de la salud en Costa Rica y en otras regiones.

\section{REFERENCIAS BIBLIOGRÁFICAS}

1. Organización Mundial de la Salud (OMS). Carta de Constitución. Ginebra: OMS; 1948.

2. Organización Panamericana de la Salud (OPS). La Renovación de la Atención Primaria de la Salud en las Américas. Documento de posición de la Organización Panamericana de la Salud/Organización Mundial de la Salud (OPS/OMS). Washington, DC: OPS; 2007.

3. Wilkinson, R; Marmot, M. Social Determinants of Health. The Solid Facts. 2th ed. Denmark: WHO; 2003.

4. Organización Mundial de la Salud (OMS).Promoción de la Salud: Glosario. Ginebra: OMS; 1998.

5. Guimarães $\mathrm{M}$, Silva $\mathrm{H}$, Noronha I. El acceso a la información como determinante social de la salud. Salud colectiva [Internet]. 2011 Oct [citado Oct 2016]; 7 (Suppl 1): S9-S18. Disponible en: http://www.scielo.org.ar/scielo.php?scrip$\mathrm{t}=$ sci_arttext\&pid=S1851-82652011000300002\&Ing=es

6. Bohórquez O, Santana A, Pérez L, Munévar, R. Seguimiento de enfermería a la madre y al recién nacido durante el puerperio: traspasando las barreras hospitalarias. Av.enferm[Serie en Internet]. 2009 Jul-Dic [citado Oct 2016]; 27 (2): 139-149. Disponible en: http://www.scielo.org.co/ pdf/aven/v27n2/v27n2a14

7. Peres W, Hilbert M. La sociedad de la información en América Latina y el Caribe: Desarrollo de las tecnologías y tecnologías para el desarrollo. CEPAL; 2009.

8. Slevin J. The Internet and Society. Unit State of America: Polity Press; 2000.

9. Organización Panamericana de la Salud (OPS). La informática y la Telemática en el campo de la salud, Usos actuales y potenciales. Washington, DC: OPS; 1990

10. Bartz C, Schlachta L, Castelli D. US Telenursing Collaborative Efforts to Achieve Goals. En: Medetel Conference. Luxembourg: Consejo Internacional de Enfermeras; 2011.p. 1-15. [Citado Feb 2016]. Disponible en: http://www.icn. ch/images/stories/documents/pillars/Practice/icnp/U.S._ Telenursing_Update.

11. Consejo Internacional de Enfermeras (CIE). Servir a la comunidad y garantizar la calidad: Las enfermeras al frente de la Atención Primaria de Salud. [Libro electrónico]. Ginebra, Suiza: CIE; 2008 [Citado Feb 2016]. Disponible en http://www.seapaonline.org/UserFiles/File/Actividades\%20de\%20Seapa/2008/DIE\%202008.pdf

12. Matiz H: El futuro de la medicina con equidad en Colombia: La Telemedicina. Revista Colombiana de Cardiología. [Revista en línea] 2007 [Citado 02 Feb 2016]; 14(1), 1-8. Disponible en http://www.scielo.org.co/scielo.
php?pid=S0120-56332007000100001\&script=sci_arttext

13. Costa I. ICN Telenursing Network. In: Congress in Technology and Humanization in Health Communication. South Africa: International Council of Nurses; 2009. p.1-18

14. Chipps J, Mars M: Technology Enhanced Learning for Remote Nurse in KeaZulu- Natala. En: IST-Africa. Durban, Sur África: IIMC International Information Management Corporation; 2010.p. 1-6. [Citado Feb 2016]. Disponible en: http://www.ist-africa.org/Conference2010/outbox/ISTAfrica_Paper_ref_95_doc_3283.pdf

15. Kouri P. Finland Interdisciplinary Telehealth. In: ICN International Conference 2011. Malta: International Council of Nurses; 2011.p.1-27.

16. Sánchez, T: Las lógicas del Telecuidado: La fabricación de la "autonomía conectada" en la teleasistencia para personas mayores [tesis doctoral]. Madrid: Universidad Autónoma de Madrid, Facultad de Filosofía y Letras; 2012.

17. Arredondo C, Siles J: Tecnología y Humanización de los Cuidados: Una mirada desde la Teoría de las Relaciones Interpersonales. Index Enferm [Revista en Internet] 2009 [Citado Feb 2016]; 18(32-36). Disponible en: http://scielo.isciii.es/scielo.php?script=sci_arttext\&pi$d=S 1132-12962009001100007$

18. Cordero M. Costa Rica es uno de los países más dinámicos en el acceso y uso de las tecnologías de la información y comunicación. El Financiero. 7 octubre 2013; Sec. Tecnología [Citado Feb 2016]; Disponible en: http://www.elfinancierocr.com/tecnologia/UIT-Internet-Tecnologias_de_Informacion_y_Comunicacion_0_386961320.html

19. Hernández R, Fernández C, Baptista P. Metodología de la Investigación. 5ta ed. México D.F: The McGraw-Hill; 2010

20. Pan American Health Organization (PAHO). Information Technology in the Health Sector of Latin America and the Caribbean: Challenges and Opportunities for the International Technical Cooperation. Washington, D.C: PAHO; 2001.

21. Programa Estado de la Nación. Decimoctavo Informe Estado de la Nación en Desarrollo Humano Sostenible. San José: Programa Estado de la Nación; 2012.

22. Gómez M. Costa Rica y el desafío hacia la Sociedad de la Información. E-Ciencia de la Información[Revista en Internet]. 2013 Ene-Jul [Citado Feb 2016]; 3(1): 1-8.Disponible en: http://revistas.ucr.ac.cr/index.php/eciencias/article/ view/8495/8018[CF6].

23. Orem D. Normas Prácticas en Enfermería. Madrid: Ediciones Pirámide, S.A; 1983.

24. Unión Internacional de Telecomunicaciones (UIT). Cumbre Mundial Sobre la Sociedad de la Información: Documentos Finales Ginebra 2003 - Túnez 2005. : Ginebra:Unión Internacional de Telecomunicaciones;2005.

25. Rojas P. Telemedicina toma fuerza en el país con iniciativas separadas a la CCSS. Crhoy. Sábado 16 de noviembre 2013.

26. González G. Evolución histórica de la enfermería informática en Costa Rica. Enfermería en Costa Rica. [Internet]. 2007 [Citado Feb 2016]; 28(1): 26-28. Disponible en: http://www.binasss.sa.cr/revistas/enfermeria/v28n1/ art5.pdf

27. Bejarano A, Sáenz M, Gutiérrez M, Alvarado R. En Las TIC en algunos de los retos del sector salud: panorama, experiencias y perspectivas. Colombia: Colombia Digital; 2003.

28. Gardner H, Davis K. La generación App: cómo los jóvenes gestionan su identidad, su privacidad y su imaginación en 
el mundo digital. Barcelona: Espasa Libro; 2014.

29. Programa Sociedad de la Información y el Conocimiento. Hacia la Sociedad de la Información y el Conocimiento. San José: Imprenta Lil; 2013.

30. Jardines J. Acceso a la Información y equidad en salud. Rev Cub Salud Pública. [Internet]. 2007 [citado Feb 2016]; 33(3): 1-8.Disponible en: http://www.redalyc.org/ pdf/214/21433309.pdf

\section{ANEXO: CUESTIONARIO UTILIZADO EN LA INVESTIGACIÓN.}

\section{CUESTIONARIO №}

Universidad de Costa Rica

Facultad de Medicina

Escuela de Enfermería

Estimada enfermera y estimado enfermero:

Se está realizando una tesis de graduación titulada "El Conocimiento, Práctica y Percepción de los enfermeros y enfermeras sobre la Tele-Enfermería como una estrategia de continuidad para el cuidado de las personas en su hogar", por lo que la recolección de la información mediante este instrumento permitirá realizar el análisis sobre este tema, el cual no ha sido abordado a nivel nacional y se considera de relevancia para el sector salud.

El cuestionario consta de cuatro partes divididas de la siguiente manera: la primera parte consta de preguntas sobre aspectos sociodemográficos, académicos y laborales, la segunda parte recoge información del conocimiento de dispositivos electrónicos, Tecnologías de la Información y la Comunicación (TIC), TIC en salud y Tele-Enfermería, la tercera parte enfoca sus preguntas a la recolección de información a fin de identificar la práctica realizada con dispositivos electrónicos, Tele-Enfermería y la realización de continuidad de cuidados en el hogar y la cuarta parte busca recopilar información sobre la percepción respecto a TIC en salud, Tele-Enfermería y continuidad de cuidados en el hogar.

Toda la información que se brinde será totalmente confidencial y anónima, y se utilizará exclusivamente con fines académicos. El cuestionario solo debe ser llenado en una ocasión y la institución en donde usted labora no tendrá acceso a los cuestionarios. Agradecemos de antemano su colaboración.

Se le adjunta el consentimiento informado que debe ser llenado previo a iniciar la aplicación del cuestionario.

Estudiantes investigadoras: Lucía Carvajal Flores lucf18@gmail. com y Lady Vázquez Vargas Ivasquez17@gmail.com .

\section{Tecnologías de la Información y la Comunicación (TIC)}

Se definen para esta investigación como "aquellas herramientas y procesos necesarios para acceder, recuperar, guardar, organizar, manipular, producir, intercambiar y presentar información por medios electrónicos. (Sunkel, 2006). Incluido el uso de teléfono fijo, móvil, ordenadores, internet, correo electrónico, chat, videoconferencia, aplicaciones telefónicas, radiodifusión, mensajes de audio y video, tabletas electrónicas y redes sociales.

\section{PARTE: Aspectos Sociodemográficos y laborales}

1. Su edad se encuentra en el siguiente rango:
( ) $18-25$
( ) $26-35$
( ) $36-45$
( ) $46-55$
( ) $56-65$
( ) Más de 65

2. ¿Cuál es su género?
( ) Femenino
( ) Masculino

3. ¿Cuál es su nacionalidad?

( ) Costarricense

( ) Otra

4. Su lugar de residencia se ubica en:

5. ¿Cuál es su grado académico?
( ) Licenciatura
( ) Maestría
( ) Doctorado
( ) Otro

6. ¿Cuál es su puesto dentro del Hospital Calderón Guardia (HCG)?

7. ¿A qué servicio pertenece usted dentro del HCG?

\section{PARTE: Conocimiento}

1. ¿Conoce cómo utilizar alguno(s) de los siguientes dispositivos electrónicos? (puede marcar más de una opción)
( ) Teléfono fijo.
( ) Teléfono Celular.
( ) Computadora.

( ) Tableta electrónica.

( ) Otro (s):

2. ¿Había escuchado sobre las Tecnologías de la Información y la Comunicación (TIC), previo a la introducción?
( ) Sí
( ) No

Si respondió que "no" pasar a pregunta No.2.4

3. ¿En dónde había escuchado sobre el tema? (Puede marcar más de una opción)
( ) En el lugar de trabajo
( ) En un congreso
( ) En un medio de prensa
( ) En la universidad
( ) Por un amigo (a)
( ) En un panfleto o brochure
( ) En internet
( ) Otro (s):

4. ¿Alguna vez ha llevado algún curso y (o) capacitación relacionado con? (Puede marcar más de una opción)
( ) Cursos en línea
( ) Computación
( ) Telemática
( ) Ninguno de los anteriores 
5. De los siguientes términos, ¿cuáles ha escuchado? (Puede marcar más de una opción)
( ) Tele-Enfermería
( ) Tele- Medicina
( ) Tele- Farmacia
( ) Tele-Cuidado
( ) Tele-Psiquiatría
( ) Tele-Salud
( ) E-health
( ) E- Learning
( ) Ninguno de los anteriores

6. ¿Conoce certeramente si en Costa Rica se utilizan Tecnologías de la Información y la Comunicación (TIC) al área de la Salud?

( ) Sí

( ) No

Si respondió que "no" pasar a pregunta No.2.8

7. ¿Dónde conoció al respecto?
( ) En el trabajo
( ) En un congreso
( ) En un medio de prensa
( ) En la universidad
( ) Por un amigo(a)
( ) En un panfleto o brochure
( ) En internet
( ) Otro (s):

8. ¿Había escuchado sobre el término Tele-Enfermería previo al cuestionario?

( ) $\mathrm{Si}$

( ) No

Si respondió que "no" pasar a pregunta No.3.1

9. ¿En dónde había escuchado sobre el tema? (Puede marcar más de una opción)
( ) En el trabajo
( ) En un congreso
( ) En un medio de prensa
( ) En la universidad
( ) Por un amigo (a)
( ) En un panfleto o brochure
( ) En internet
( ) Otro (s):

\section{PARTE: Práctica}

1. Mencione cuál (es) de los siguientes dispositivos electrónicos tiene usted (Puede marcar más de una opción)
( ) Teléfono fijo.
( ) Celular.
( ) Computadora.
( ) Tableta electrónica.
( ) Otro (s):

2. ¿Utiliza alguna (s) de las siguientes aplicaciones en su dispositivo electrónico? (Puede marcar más de una opción)
( ) Educación
( ) Libros
( ) Negocios
( ) Entretenimiento
( ) Finanzas
( ) Comidas y bebidas
( ) Salud y forma

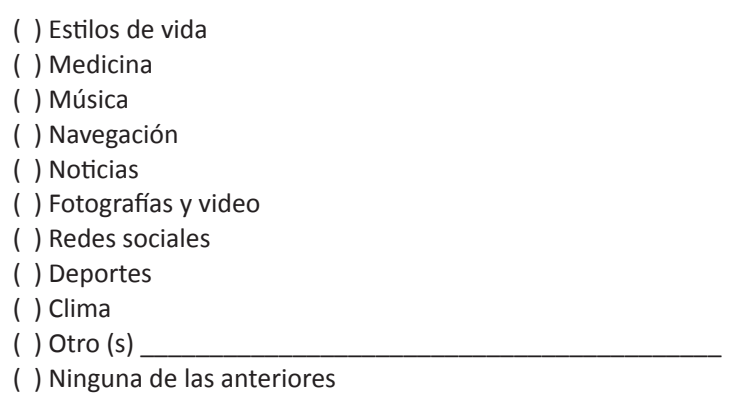

3. ¿Utiliza el teléfono fijo de su lugar de trabajo?

( ) Sí

( ) No

Si respondió que "no" pasar a pregunta No.3.5

4. ¿Con qué fines utiliza el teléfono fijo en su lugar de trabajo? (Puede marcar más de una opción)

( ) Para comunicarse con algún otro servicio y/o departamento.

( ) Para conocer resultados de laboratorios y exámenes médicos.

( ) Para comunicarse con usuarios o usuarias respecto al estado de salud.

( ) Para comunicarse con la familia o responsables de un usuario o usuaria.

( ) Para comunicarse con otros profesionales del área de la salud.

( ) Para localizar expedientes.

( ) Otro (s):

5. ¿Tiene computadora en su lugar de trabajo?
( ) Sí
( ) No
Si respondió que "no" pasar a pregunta No.3.9

6. ¿Utiliza la computadora en su lugar de trabajo?

( ) Sí

( ) No

Si respondió que "no" pasar a pregunta No.3.8

Si respondió que "si" avanzar de la 3.7 a la 3.9

7. ¿Con qué fines utiliza la computadora de su lugar de trabajo? (Puede marcar más de una opción)

( ) Para comunicarse con algún otro servicio y/o departamento.

( ) Para conocer resultados de laboratorios y exámenes médicos.

( ) Para comunicarse con usuarios o usuarias respecto al estado de salud.

( ) Para comunicarse con la familia o responsables de un usuario o usuaria.

( ) Para comunicarse con otros profesionales del área de la salud.

( ) Para localizar expedientes.

( ) Otro (s):

8. ¿Por qué motivo no utiliza la computadora en su lugar de trabajo? (Puede marcar más de una opción).

( ) Desconoce cómo utilizarla

( ) Pasa ocupada por otros profesionales

( ) No se le permite utilizarla

( ) La computadora trabaja muy lento 
( ) No tiene tiempo

( ) No la requiere en sus horas laborales

( ) Otro (s):

( ) Ninguno/NAR

9. Para establecer comunicación e informarse sobre un usuario o usuaria a distancia ¿usted ha utilizado alguno de los siguientes medios? (Puede marcar más de una opción)

( ) Una llamada telefónica

( ) Una video llamada

( ) Mensajes de texto

( ) Un Chat

( ) Un correo electrónico

( ) Otro (s):

( ) Ninguno/NAR

10. Con el fin de dar continuidad de cuidados en el hogar a un usuario o usuaria a distancia, ¿usted ha utilizado alguno de los dispositivos electrónicos? (Puede marcar más de una opción)

( ) Teléfono fijo.

( ) Celular.

( ) Computadora.

( ) Tableta electrónica.

( ) Otro (s):

( ) Ninguno de los anteriores

Si respondió que "ninguno de los anteriores" pasar a pregunta No.4.1

11. ¿Usted ha utilizado alguna de las siguientes herramientas didácticas, con el fin de dar continuidad de cuidados en el hogar, a un usuario o usuaria que se encuentra a la distancia? (Puede marcar más de una opción)

( ) Presentación en Power Point o programas semejantes

( ) Correo electrónico informativo

( ) Videos interactivos

( ) Brochures informativos

( ) Mensajes de audio

( ) Otro (s):

( ) Ninguno/NAR

\section{PARTE:Percepción}

1. ¿Qué opina usted sobre el uso de Tecnologías de la Información y la Comunicación (TIC)? (solo una opción)

( ) Considera que son útiles y necesarias.

( ) Considera que son útiles pero no necesarias.

( ) Considera que no son útiles ni necesarias.

2. ¿Cree que las Tecnologías de la Información y la Comunicación (TIC) son una herramienta funcional para el campo de la Salud?

( ) Totalmente de acuerdo.

( ) De acuerdo.

( ) Ni de acuerdo ni en desacuerdo.

( ) En desacuerdo.

( ) Totalmente en desacuerdo.

3. ¿Considera que las Tecnologías de la Información y la Comunicación (TIC) se aplican actualmente a la Enfermería en Costa Rica?

( ) Totalmente de acuerdo.

( ) De acuerdo.

( ) Ni de acuerdo ni en desacuerdo.

( ) En desacuerdo.

( ) Totalmente en desacuerdo.
4. ¿Considera importante tener acceso a los dispositivos electrónicos de su lugar de trabajo para efectos laborales?
( ) Totalmente de acuerdo.
( ) De acuerdo.
( ) Ni de acuerdo ni en desacuerdo.
( ) En desacuerdo.
( ) Totalmente en desacuerdo.

5. ¿Cree que el desarrollo de las Tecnologías de la Información y la Comunicación pueden aplicarse a la Enfermería?

( ) Totalmente de acuerdo.

( ) De acuerdo.

( ) Ni de acuerdo ni en desacuerdo.

( ) En desacuerdo.

( ) Totalmente en desacuerdo.

6. ¿Considera que brindar servicios de Enfermería a la distancia es posible en Costa Rica?

( ) Totalmente de acuerdo.

( ) De acuerdo.

( ) Ni de acuerdo ni en desacuerdo.

( ) En desacuerdo.

( ) Totalmente en desacuerdo.

7. ¿Considera que la Enfermería puede dar continuidad de cuidados en el hogar a la distancia por medio de dispositivos electrónicos?

( ) Totalmente de acuerdo.

( ) De acuerdo.

( ) Ni de acuerdo ni en desacuerdo.

( ) En desacuerdo.

( ) Totalmente en desacuerdo.

8. ¿Considera que los cuidados a distancia despersonalizaría la atención de Enfermería?

( ) Totalmente de acuerdo.

( ) De acuerdo.

( ) Ni de acuerdo ni en desacuerdo.

( ) En desacuerdo.

( ) Totalmente en desacuerdo.

9. ¿Opina que dentro de su lugar de trabajo, sería viable la implementación de la Tele-Enfermería como una estrategia de continuidad para el cuidado de las personas en su hogar?

( ) Totalmente de acuerdo.

( ) De acuerdo.

( ) Ni de acuerdo ni en desacuerdo.

( ) En desacuerdo.

( ) Totalmente en desacuerdo.

10. ¿Estaría dispuesto(a) a brindar continuidad de cuidados en el hogar por medio de Tele-Enfermería?

( ) Totalmente de acuerdo.

( ) De acuerdo.

( ) Ni de acuerdo ni en desacuerdo.

( ) En desacuerdo.

( ) Totalmente en desacuerdo.

11. ¿Le gustaría capacitarse sobre Tele-Enfermería?

( ) Sí

( ) No

¡Muchas gracias por su colaboración! 